\title{
Effects of Glucose Abnormalities on In-Hospital Outcome After Coronary Intervention for Acute Myocardial Infarction
}

\author{
Masami Kosuge, MD; Kazuo Kimura, MD; Sunao Kojima, MD*; Tomohiro Sakamoto, MD*; \\ Kunihiko Matsui, MD*; Masaharu Ishihara, MD**; Yujiro Asada, MD ${ }^{\dagger}$; Chuwa Tei, MD ${ }^{\dagger \dagger}$; \\ Shunichi Miyazaki, MD\$; Masahiro Sonoda, MD\$; Kazufumi Tsuchihashi, MD\&; \\ Masakazu Yamagishi, MD; Yoshihiko Ikeda, MD§§; Mutsunori Shirai, MD***; \\ Hisatoyo Hiraoka, MD ${ }^{\dagger}$; Takeshi Inoue, MD $\$$; Fumio Saito, MD\$§§; Hisao Ogawa, MD* \\ on behalf of the Japanese Acute Coronary Syndrome Study (JACSS) Investigators
}

\begin{abstract}
Background The effects of glucose abnormalities on outcomes after percutaneous coronary intervention (PCI) remain unclear. We examined the association between glucose abnormalities and in-hospital outcome in patients undergoing PCI for acute myocardial infarction (AMI).

Methods and Results A total of 849 patients with AMI who were admitted within $12 \mathrm{~h}$ after symptom onset and underwent emergency PCI were classified according to the presence or absence of admission hyperglycemia, defined as a blood glucose level on admission of $>11 \mathrm{mmol} / \mathrm{L}$ and whether they had a history of diabetes mellitus: group $1(\mathrm{n}=504)$, non-diabetic patients without admission hyperglycemia; group $2(\mathrm{n}=111)$, diabetic patients without admission hyperglycemia; group $3(\mathrm{n}=87)$, non-diabetic patients with admission hyperglycemia; and group $4(\mathrm{n}=147)$, diabetic patients with admission hyperglycemia. Among groups 1, 2, 3 and 4, in-hospital mortality was $2.6,2.7,11.5$ and $8.8 \%$, respectively $(\mathrm{p}<0.01)$. Multivariate analysis showed that compared with group 1 patients, the odds ratio (95\% confidence interval) for in-hospital mortality among those in groups 2,3 , and 4 were $0.80(0.24-2.60, \mathrm{p}=0.708), 2.29(1.10-5.49, \mathrm{p}=0.039)$, and $2.14(1.14-4.69, \mathrm{p}=0.048)$, respectively.

Conclusions In-patients undergoing PCI for AMI, admission hyperglycemia, irrespective of the presence or absence of diabetes, is associated with increased in-hospital mortality, whereas diabetes without admission hyperglycemia is not. (Circ J 2005; 69: 375-379)
\end{abstract}

Key Words: Glucose; Myocardial infarction; Reperfusion; Stent

$\mathbf{P}$ atients with diabetes have been established to have poorer outcomes after acute myocardial infarction (AMI) than non-diabetic patients!,2 Furthermore, hyperglycemia itself on admission (admission hyperglycemia) is also associated with an increased risk of adverse

(Received November 1, 2004; revised manuscript received December 22, 2004; accepted January 11, 2005)

Division of Cardiology, Yokohama City University Medical Center, Yokohama, *Department of Cardiovascular Medicine, Graduate School of Medical Sciences, Kumamoto University, Kurume, **Department of Cardiology, Hiroshima City Hospital, Hiroshima, ${ }^{\dagger}$ First Department of Pathology, Miyazaki University School of Medicine, Miyazaki, the First Department of Internal Medicine, Kagoshima University Faculty of Medicine, Kagoshima, "Division of Cardiology, Department of Internal Medicine, National Cardiovascular Center, Suita, $¥$ The Second Department of Cardiology, National Hospital Kyusyu Cardiovascular Center, Kagoshima, \$Second Department of Internal Medicine, Sapporo Medical University School of Medicine, Sapporo, $\$ \$$ Department of Pathology, National Cardiovascular Center, Suita, ***Department of Microbiology, School of Medicine, Yamaguchi University, Ube, Hepartment of Internal Medicine and Molecular Science, Graduate School of Medicine, Osaka University, Suita, \#ivision of Cardiology, Oita National Hospital, Oita and $\S \$$ Department of Cardiology, Nihon University Surugadai Hospital, Tokyo, Japan

Mailing address: Kazuo Kimura, MD, The Division of Cardiology, Yokohama City University Medical Center, 4-57 Urafune-cho, Minami-ku, Yokohama 232-0024, Japan. E-mail: c-kimura@urahp. yokohama-cu.ac.jp events, including heart failure, cardiogenic shock, and death after AMI, irrespective of whether diabetes was previously diagnosed ${ }^{3-6}$ Recently, Wahab et al report that diabetes, admission hyperglycemia, or both were associated with adverse outcomes after AMI during the thrombolytic era6

Thrombolytic therapy has been established to significantly reduce mortality among both diabetic and non-diabetic patients with AMI? Despite substantial benefits, thrombolytic therapy is less likely to be given to diabetic patients, which might contribute to their poorer outcome ${ }^{6,8}$ Recently, percutaneous coronary intervention (PCI) is increasingly used for reperfusion therapy, improving the outcome of patients with AMI. In diabetic patients with AMI, primary angioplasty is associated with fewer and less severe adverse events than thrombolytic therapy, suggesting that PCI might have a beneficial effect on survival in diabetic patients. The aim of this study was to examine the relations of glucose abnormalities to infarct size and in-hospital mortality in patients with AMI who underwent PCI.

\section{Methods}

\section{Study Population}

The Japan Acute Coronary Syndrome Study (JACSS) was a retrospective, observational multicenter trial. Between January and December 2001, patients with AMI admitted to 35 participating hospitals in Japan were studied. 
Table 1 Baseline Characteristics

\begin{tabular}{|c|c|c|c|c|c|}
\hline & $\begin{array}{l}\text { Group 1 } \\
(n=504)\end{array}$ & $\begin{array}{l}\text { Group } 2 \\
(n=111)\end{array}$ & $\begin{array}{c}\text { Group } 3 \\
(n=87)\end{array}$ & $\begin{array}{l}\text { Group } 4 \\
(n=147)\end{array}$ & $p$ value \\
\hline Age (years) & $65 \pm 12$ & $66 \pm 10$ & $71 \pm 12$ & $66 \pm 13$ & $<0.01$ \\
\hline Male & $77 \%$ & $79 \%$ & $68 \%$ & $65 \%$ & $<0.01$ \\
\hline Time from symptom onset to admission $(h)$ & $3.6 \pm 2.8$ & $3.2 \pm 2.4$ & $3.0 \pm 2.2$ & $3.3 \pm 2.5$ & NS \\
\hline Killip $>1$ on admision & $14 \%$ & $14 \%$ & $25 \%$ & $27 \%$ & $<0.01$ \\
\hline Previous infarction & $10 \%$ & $18 \%$ & $10 \%$ & $14 \%$ & NS \\
\hline Previous angina & $40 \%$ & $40 \%$ & $38 \%$ & $29 \%$ & $N S$ \\
\hline Blood glucose level on admission $(\mathrm{mmol} / \mathrm{L})$ & $7.5 \pm 1.7$ & $8.4 \pm 1.7$ & $13.9 \pm 3.2$ & $16.1 \pm 4.6$ & $<0.01$ \\
\hline $\operatorname{HbAlc}(\%)^{\dagger}$ & $\begin{array}{l}5.3 \pm 0.6 \\
(n=209)\end{array}$ & $\begin{array}{l}6.9 \pm 1.2 \\
(n=78)\end{array}$ & $\begin{array}{l}5.7 \pm 0.9 \\
(n=31)\end{array}$ & $\begin{array}{c}8.1 \pm 1.8 \\
(n=102)\end{array}$ & $<0.01$ \\
\hline Diabetes mellitus & 0 & $100 \%$ & 0 & $100 \%$ & \\
\hline Hyperlipidemia & $28 \%$ & $45 \%$ & $23 \%$ & $41 \%$ & $<0.01$ \\
\hline Hypertension & $53 \%$ & $63 \%$ & $53 \%$ & $62 \%$ & NS \\
\hline Smoking & $52 \%$ & $55 \%$ & $45 \%$ & $46 \%$ & NS \\
\hline Serum creatinine on admission $(\mathrm{mg} / \mathrm{d})$ & $0.9 \pm 0.9$ & $1.1 \pm 1.1$ & $1.0 \pm 0.8$ & $1.2 \pm 1.2$ & NS \\
\hline \multicolumn{6}{|l|}{ Medication before AMI } \\
\hline Oral hypoglycemic drug & 0 & $35 \%$ & 0 & $45 \%$ & $<0.01$ \\
\hline Insulim & 0 & $9 \%$ & 0 & $22 \%$ & $<0.01$ \\
\hline Aspirin & $8 \%$ & $16 \%$ & $7 \%$ & $15 \%$ & $<0.01$ \\
\hline$\beta$-blocker & $4 \%$ & $7 \%$ & $6 \%$ & $5 \%$ & NS \\
\hline ACE inhibitor & $5 \%$ & $12 \%$ & $6 \%$ & $10 \%$ & $<0.05$ \\
\hline$H M G C O A$ & $4 \%$ & $15 \%$ & $7 \%$ & $14 \%$ & $<0.01$ \\
\hline Anterior AMI & $51 \%$ & $40 \%$ & $56 \%$ & $43 \%$ & $N S$ \\
\hline ST-segment elevation & $91 \%$ & $90 \%$ & $95 \%$ & $91 \%$ & $N S$ \\
\hline 3-vessel disease & $10 \%$ & $19 \%$ & $12 \%$ & $21 \%$ & $<0.01$ \\
\hline TIMI flow grade 0 at initial CAG & $68 \%$ & $61 \%$ & $75 \%$ & $63 \%$ & $N S$ \\
\hline Final TIMI flow grade $\geq 2$ & $97 \%$ & $97 \%$ & $97 \%$ & $95 \%$ & NS \\
\hline Final TIMI flow grade 3 & $90 \%$ & $87 \%$ & $87 \%$ & $88 \%$ & $N S$ \\
\hline Stent implantation & $79 \%$ & $73 \%$ & $83 \%$ & $78 \%$ & NS \\
\hline
\end{tabular}

AMI, acute myocardial infarction; ACE, angiotensin-converting enzyme; CAG, coronary angiography; HbAIc, glycosylated homoglobin; HMG CoA, hydroxymethyIglutaryl-coenzyme A reductase inhibitors.

HbAlc was measured during hospitalization in only 420 patients.

Group 1, Non-diabetic patients without admission hyperglycemia; Group 2, Diabetic patients without admission hyperglycemia;

Group 3, Non-diabetic patients with admission hyperglycemia; Group 4, Daibetic patients with admission hyperglycemia.

Data are presented as mean values $\pm S D$ or percentages of patients.

A diagnosis of AMI required at least 2 of the following characteristics: typical chest pain persisting for $30 \mathrm{~min}$ or longer, ischemic electrocardiographic changes, and a peak creatine kinase level equivalent to more than twice the upper limit of normal. The study protocol was reviewed and approved by the ethical committee of each participating hospital. A total of 849 patients who met the following entry criteria were studied: (i) admission within $12 \mathrm{~h}$ from the onset of AMI; (ii) coronary angiography performed immediately after admission; (iii) percutaneous transluminal coronary angioplasty, stenting, or both of the infarctrelated artery; (iv) measurement of blood glucose level on admission; and (v) availability of a detailed clinical history. Data from all subjects, excluding information that could be used to identify patients, such as names and identification numbers, were transmitted to a central data collection center, located in the Department of Cardiovascular Medicine, Graduate School of Medical Sciences, Kumamoto University, for analysis.

\section{Coronary Angiography and Coronary Intervention}

Written informed consent for coronary catheterization was obtained from all patients at each hospital. Coronary angiography was performed immediately after admission. The perfusion status of the infarct-related artery was assessed according to the Thrombolysis in Myocardial Infarction (TIMI) study classification $!^{0}$ The recanalization method was left to the physicians' discretion. Final TIMI flow grade was assessed on the basis of final angiograms obtained on admission.

\section{Data Analysis}

Previous angina was defined as the presence of typical chest pain occurring at rest or during exercise and persisting for less than $30 \mathrm{~min}$, within $24 \mathrm{~h}$ before the onset of AMI. Diabetes mellitus was considered present if this diagnosis and antidiabetic treatment, including drugs or insulin, had been given to the patient, if the fasting glucose level was found to $\mathrm{be}=126 \mathrm{mg} / \mathrm{dl}(7.0 \mathrm{mmol} / \mathrm{L})$ on the previous occasion or if the results of an oral glucose tolerance test were abnormal. Patients who did not meet these criteria were considered not to have diabetes mellitus. Blood samples for measurement of blood glucose level were obtained on admission. Admission hyperglycemia was defined as a blood glucose level on admission of $>198 \mathrm{mg} / \mathrm{dl}(11 \mathrm{mmol} / \mathrm{L})^{6}, 11$ Glycosylated hemoglobin (HbA1c) was measured in 420 patients (49\%) within 14 days after admission. Patients were classified into 4 groups, based on their history of diabetes and their blood glucose level on admission:

Group 1 (n=504): Non-diabetic patients without admission hyperglycemia;

Group $2(\mathrm{n}=111)$ : Diabetic patients without admission hyperglycemia;

Group $3(n=87)$ Non-diabetic patients with admission hyperglycemia;

Group $4(\mathrm{n}=147)$ : Diabetic patients with admission hyperglycemia. 


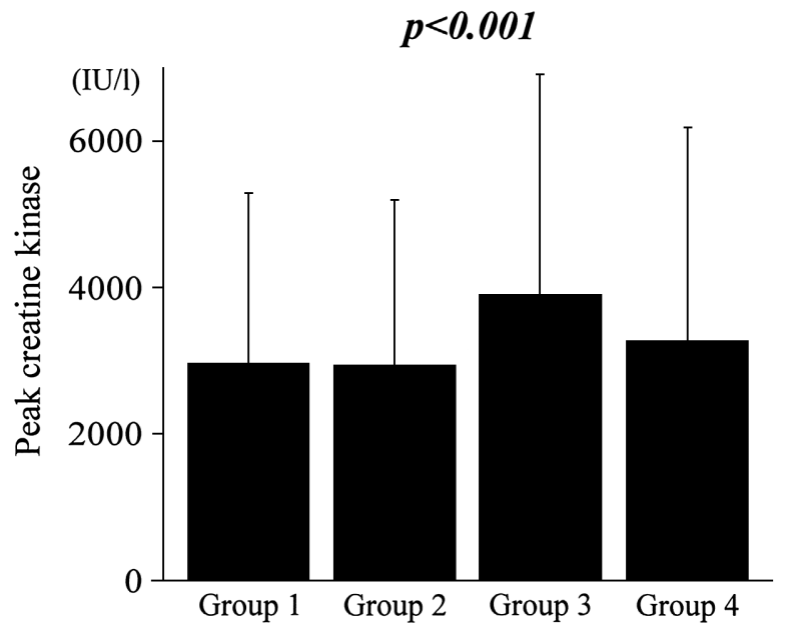

Fig 1. Peak creatine kinase level was higher in group 3, and infarct sizes were similar in the other 3 groups.

\section{Statistical Analysis}

Data are expressed as mean values \pm standard deviation for continuous variables and as percentages for categorical variables. We made comparisons by one-way analysis of variance for continuous variables, and the statistical significance of differences was calculated by using the Scheffe F test. Chi-squared analysis or Fisher's exact test was used to compare categorical variables. A two-tailed $\mathrm{p}$ value of $<0.05$ was considered to indicate statistical significance. Multiple logistic regression analysis was used to examine determinants of in-hospital mortality. Variables used for analysis included an age of $>70$ years, ${ }^{12}$ sex, time to admission, Killip $>1$ on admission, previous infarction, serum creatinine level on admission, ST-segment elevation, anterior infarction, absence of previous angina within $24 \mathrm{~h}$ before symptom onset, occlusion status at the culprit lesion, 3-vessel disease, stent implantation, final TIMI flow grade $\leq 2$, and glycemic status. The strength of association of glycemic status was assessed by comparison of the 3 groups with a disordered blood glucose profile to the normal (group 1) patients who had no diagnosis of diabetes without admission hyperglycemia. Analyses were conducted with the use of SPSS PC software.

\section{Results}

\section{Patient Characteristics}

The overall prevalence of diabetes in the study group was $30 \%$. Patients' characteristics in the 4 study subgroups are presented in Table 1. Non-diabetic patients with admission hyperglycemia were likely to be oldest. Patients with admission hyperglycemia were likely to be female and to be in the Killip class $>1$ on admission, and independent of a diabetic status. The prevalence of previous infarction was slightly but not significantly higher in the diabetic patients than in the non-diabetic patients. Diabetic patients with admission hyperglycemia had the highest blood glucose level on admission and the highest $\mathrm{HbA}_{1 \mathrm{C}}$ value. In general, diabetic patients were more likely to have hyperlipidemia and hypertension than non-diabetic patients. Diabetic patients were more likely to be receiving aspirin, angiotensin-converting enzyme inhibitors, and hydroxymethylglutarylcoenzyme A reductase inhibitors. There were no differences in the 4 groups with regard to time from symptom onset to

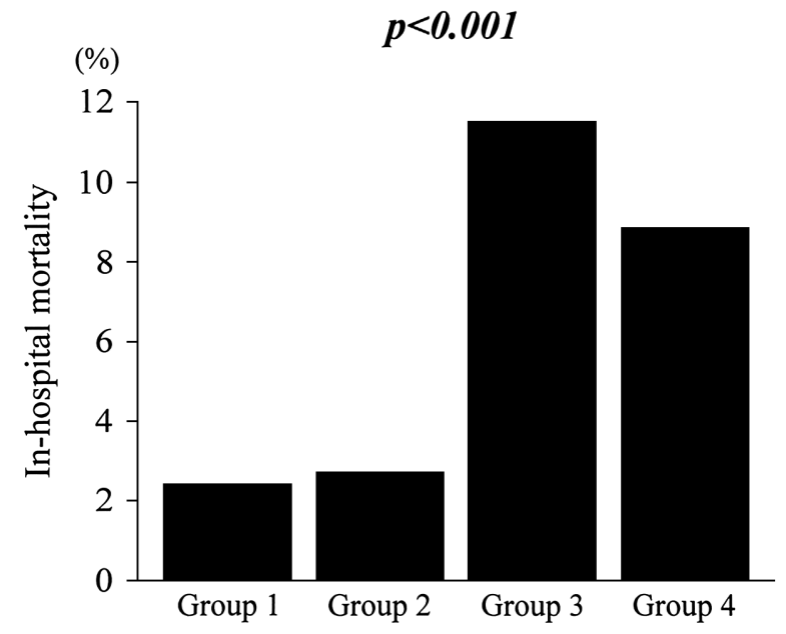

Fig 2. In-hospital mortality was highest in group 3 and second highest in group 4.

admission, infarct location, serum creatinine level on admission, and prevalence of ST-segment elevation.

\section{Coronary Angiographic Findings}

The coronary angiographic findings of the patients are presented in Table 1. Stent implantation was performed in 665 patients $(78 \%)$. Diabetic patients were more likely to have 3 -vessel disease than non-diabetic patients. There were no significant differences in the 4 groups with respect to the prevalences of TIMI flow grade 0 at initial coronary angiography, a final TIMI flow grade $\geq 2$, a final TIMI flow grade of 3 , or stent implantation.

\section{Peak Creatine Kinase Level}

Non-diabetic patients with admission hyperglycemia had a higher peak creatine kinase level than the other 3 groups, which had similar levels (Fig 1).

\section{In-Hospital Mortality}

During hospitalization (mean 14 days), 39 patients (4.5\%) died (38 of cardiac causes and one of multiple organ failure). In-hospital mortality was higher in non-diabetic and diabetic patients with admission hyperglycemia, especially in the former (Fig 2). Multivariate analysis showed that patients who were $>70$ years of age, had Killip $>1$ on admission, serum creatinine on admission, anterior infarction, final TIMI grade $\leq 2$, and admission hyperglycemia, irrespective of the presence or absence of diabetes (groups 3 and 4), were independent predictors of in-hospital death (Table 2).

\section{Discussion}

Our findings suggest that in-patients undergoing PCI for $\mathrm{AMI}$, and the presence of admission hyperglycemia with or without diabetes significantly contributed to in-hospital mortality. Diabetes without admission hyperglycemia did not increase in-hospital mortality.

\section{Non-Diabetic Patients With Admission Hyperglycemia}

The poor outcome in non-diabetic patients with admission hyperglycemia may arise from a larger infarct size. Hyperglycemia has been shown to increase intercellular adhesion molecule-1, which increases the leukocyte plug- 
Table 2 Multivariate Analysis of Factors Associated With In-Hospital Mortality

\begin{tabular}{lcc}
\hline \hline Variable & Odds ratio $(95 \%$ CI) & $p$ value \\
\hline Group 1 & $1.00(-)$ & - \\
Group 2 & $0.80(0.24-2.60)$ & 0.708 \\
Group 3 & $2.29(1.10-5.49)$ & 0.039 \\
Group 4 & $2.14(1.14-4.69)$ & 0.048 \\
Age $>70$ years & $3.09(1.08-9.81)$ & 0.049 \\
Sex & $0.90(0.32-2.55)$ & 0.636 \\
Time to admission & $1.07(0.75-1.16)$ & 0.519 \\
Killip $>$ 1 on admission & $5.49(1.88-16.0)$ & 0.002 \\
Previous infarction & $2.49(0.73-8.45)$ & 0.143 \\
Serum creatinine on admission & $1.82(1.13-2.93)$ & 0.014 \\
ST-segment elevation & $0.49(0.11-2.29)$ & 0.336 \\
Anterior infarction & $5.45(1.68-17.7)$ & 0.005 \\
Absence of previous angina & $1.15(0.37-3.64)$ & 0.280 \\
TIMI flow grade 0 at initial CAG & $3.67(0.96-14.0)$ & 0.057 \\
3-vessel disease & $2.93(0.95-8.98)$ & 0.061 \\
Stent implantation & $1.10(0.32-3.02)$ & 0.976 \\
Final TIMI flow grade $\leq 2$ & $3.53(1.06-11.7)$ & 0.039 \\
\hline
\end{tabular}

CAG, coronary angiography.

Group 1, Non-diabetic patients without admission hyperglycemia; Group 2, Diabetic patients without admission hyperglycemia; Group 3, Non-diabetic patients with admission hyperglycemia; Group 4, Daibetic patients with admission hyperglycemia.

ging of capillaries, ${ }^{13}$ augments platelet-dependent thrombus formation, 14 and attenuates endothelium-dependent vasodilation 15 Although these mechanisms may contribute to a larger infarct size, we cannot rule out the possibility that hyperglycemia was caused by severe myocardial damage 16 The poor outcome in non-diabetic patients with admission hyperglycemia might also be related to undiagnosed diabetes. Unrecognized diabetes or impaired glucose tolerance may increase endothelial damage due to untreated glucose abnormalities. Two recent studies show that abnormal glucose metabolism is very common in patients with AMI: approximately two-thirds of patients with no previous diagnosis of diabetes have undetected diabetes or impaired glucose tolerance! ${ }^{17,18}$

\section{Diabetic Patients With or Without Admission Hyperglycemia}

Diabetic patients with admission hyperglycemia had a very high blood glucose level on admission. Nonetheless, these patients had a relatively small infarct size, similar to those in patients without admission hyperglycemia. Diabetic patients with admission hyperglycemia had a higher rate of insulin treatment and a higher $\mathrm{HbA} 1 \mathrm{C}$ value, suggesting a longer duration of severe diabetes. Diabetic patients who have impaired islet responses to glucose, especially those with insulin-dependent diabetes, are particularly prone to the development of marked hyperglycemia during stress states ${ }^{19,20}$ Marked hyperglycemia in these patients may therefore not correlate with infarct size. However, diabetic patients with admission hyperglycemia had higher inhospital mortality than did patients in the other groups without admission hyperglycemia. Our findings suggest that the poor outcome in diabetic patients with admission hyperglycemia is primarily related to the deleterious effects of diabetes on myocardial function rather than to infarct size. One explanation is the existence of a specific form of heart muscle disease associated with diabetes. Clinically, this disease manifests itself as left ventricular dysfunction or failure. ${ }^{1}$ The higher prevalence of Killip class $>1$ on admission in diabetic patients with admission hyperglycemia, despite a similar infarct size as compared with patients without admission hyperglycemia, may reflect increased susceptibility to the deleterious effects of diabetes. Such effects might be most obvious in patients with a prolonged history of severe diabetes. Hyperglycemia itself may directly impair left ventricular function6 Furthermore, poorly controlled diabetes may relate to microvascular dysfunction?22 Moreover, coronary atherosclerosis may be more severe and diffuse in diabetic patients with admission hyperglycemia, 23 as indicated by the higher incidence of 3 -vessel disease. Severer ischemia in the non-infarcted myocardium might increase the risk of heart failure.

Diabetic patients without admission hyperglycemia had a smaller infarct size and a better in-hospital outcome than did patients with admission hyperglycemia, regardless of whether they had a history of diabetes. These findings do not support the results of a recent study by Wahab et al, who showed that diabetic patients, irrespective of admission hyperglycemia, have higher mortality after AMI than non-diabetic patients. In-hospital mortality in their diabetic patients was much higher than that in the patients of the present study. These disparate findings may relate to the different treatment strategies used. Patients in the study by Wahab et al, especially those who were diabetic, were less likely to receive thrombolysis or PCI. The worse outcome in their diabetic patients might thus be related, at least in part, to inadequate reperfusion therapy, as suggested previously, 6,8 In contrast, we studied only patients who received PCI, and our final success rate was high. The better inhospital outcome of diabetic patients without admission hyperglycemia in the present study suggests that a higher rate of reperfusion by PCI might improve survival in such patients, compared with that of previous studies. Another important distinction between the 2 studies involves the baseline characteristics of diabetic patients without admission hyperglycemia. In the present study, a smaller proportion of patients were receiving insulin treatment, and the mean $\mathrm{HbA} 1 \mathrm{C}$ value was $6.9 \%$, suggesting relatively good glycemic control. Our subjects most likely had milder or a shorter duration of diabetes than those studied by Wahab et al6 Experimental studies have shown that the heart in the early phase of diabetes is more resistant to ischemia than the non-diabetic heart ${ }^{24}$ Another study has reported that a shorter duration of diabetes is associated with a better outcome after AMI! These findings suggest that the duration and severity of diabetes are important determinants of outcome.

\section{Study Limitations}

This was a retrospective, observational and non-randomized study. However, we included approximately two-thirds of all patients who were admitted to JACSS-affiliated hospitals within $12 \mathrm{~h}$ from the onset of AMI. Therefore, we believe that our results serve to demonstrate the effect of glucose abnormalities on in-hospital outcome in patients who receive PCI. In the present study, diabetes mellitus was diagnosed on the basis of whether patients were receiving antidiabetic treatment, blood glucose levels were measured before admission, and the results of oral glucose tolerance tests were available. However, diabetes may have not been diagnosed with the use of these general criteria in some "non-diabetic" patients. The inclusion of such patients may have substantially affected the study results. The inability to exclude such patients from this multicenter retrospective investigation represents an important limitation of our 
study design. Nonetheless, the proportion of our subjects who had diabetes (approximately 30\%) was consistent with that of previous studies of patients. 5 Furthermore, we evaluated infarct size on the basis of peak creatine kinase level, but peak creatine kinase level may not accurately reflect infarct size. Other techniques that allow direct examination of infarct size, such as radioisotopes, are needed to more objectively evaluate infarct size and provide important additional information. Further prospective studies involving larger numbers of patients are required to confirm the effect of admission hyperglycemia for patients on outcome after PCI for AMI.

\section{Conclusions}

Our findings suggest that in-patients undergoing PCI for AMI, and admission hyperglycemia, irrespective of the presence or absence of diabetes, is associated with increased in-hospital mortality, whereas diabetes without admission hyperglycemia is not.

\section{Acknowledgments}

The authors gratefully acknowledge that the present study was supported by a Research Grant for Cardiovascular Disease (14C-4) from the Ministry of Health, Labour and Welfare.

\section{References}

1. Barbash GI, White HD, Modan M, Van de Werf F. Significance of diabetes mellitus in patients with acute myocardial infarction receiving thrombolytic therapy: Investigators of the International Tissue Plasminogen Activator/Streptokinase Mortality Trial. J Am Coll Cardiol 1993; 22: 707-713.

2. Mak KH, Moliterno DJ, Granger CB, Miller DP, White HD, Wilcox $\mathrm{RG}$, et al. Influence of diabetes mellitus on clinical outcome in the thrombolytic era of acute myocardial infarction: GUSTO-I Investigators: Global Utilization of Streptokinase and Tissue Plasminogen Activator for Occluded Coronary Arteries. J Am Coll Cardiol 1997; 30: $171-179$.

3. Oswald GA, Corcoran S, Yudkin JS. Prevalence and risk of hyperglycemia and undiagnosed diabetes in patients with acute myocardial infarction. Lancet 1984; 1: 1264-1267.

4. Fava S, Aquilina O, Azzopardi J, Agius Muscat H, Fenech FF. The prognostic value of blood glucose in diabetic patients with acute myocardial infarction. Diabetic Med 1996; 13: 80-83

5. Capes SE, Hunt D, Malmberg K, Gerstein HC. Stress hyperglycemia and increased risk after myocardial infarction in patients with and without diabetes: A systematic overview. Lancet 2000; 355: $773-$ 778.

6. Wahab NN, Cowden EA, Pearce NJ, Gardner MJ, Merry H, Cox JL, et al. Is blood glucose an independent predictor of mortality in acute myocardial infarction in the thrombolytic era? J Am Coll Cardiol 2002; 40: $1748-1754$

7. Fibrinolytic Therapy Trialists' (FTT) Collaborative Group. Indications for fibrinolytic therapy in suspected acute myocardial infarction: Collaborative overview of early mortality and major morbidity results from all randomised trials of more than 1000 patients. Fibrinolytic Therapy Trialists' (FTT) Collaborative Group. Lancet 1994; 343: $311-322$.

8. Pfeffer MA, Moye LA, Braunwald E, Basta L, Brown EJ Jr, Cuddy TE, et al. Selection bias in the use of thrombolytic therapy in acute myocardial infarction: The SAVE Investigators. JAMA 1991; 266: 528-532.

9. Hsu LF, Mak KH, Lau KW, Sim LL, Chan C, Koh TH, et al. Clinical outcomes of patients with diabetes mellitus and acute myocardial infarction treated with primary angioplasty or fibrinolysis. Heart 2002; 88: 260-265.

10. The TIMI study group. The Thrombolysis in Myocardial Infarction (TIMI) trial: Phase I findings. N Engl J Med 1985; 312: 932-936.

11. Report of the Expert Committee on the Diagnosis and Classification of Diabetes Mellitus. American Diabetes Association: Clinical practice recommendation 2002. Diabetes Care 2002; 25: S1-S147.

12. The TIMI Study Group. Comparison of invasive and conservative strategies after treatment with intravenous tissue plasminogen activa- tor in acute myocardial infarction: Results of the thrombolysis in myocardial infarction (TIMI) phase II trial. N Engl J Med 1989; 320: $618-627$.

13. Hokama JY, Ritter LS, Davis-Gorman G, Cimetta AD, Copeland JG, McDonagh PF. Diabetes enhances leukocyte accumulation in the coronary microcirculation early in reperfusion following ischemia. $J$ Diabetes Complications 2000; 14: 96-107.

14. Shechter M, Merz CN, Paul-Labrador MJ, Kaul S. Blood glucose and platelet-dependent thrombosis in patients with coronary artery disease. J Am Coll Cardiol 2000; 35: 300-307.

15. Title LM, Cummings PM, Giddens K, Nassar BA. Oral glucose loading acutely attenuates endothelium-dependent vasodilation in healthy adults without diabetes: An effect prevented by vitamins $\mathrm{C}$ and E. J Am Coll Cardiol 2000; 36: 2185-2191.

16. Oswald GA, Smith CCT, Betteridge DJ, Yudkin JS. Determinants and importance of stress hyperglycemia in non-diabetic with myocardial infarction. Br Med J 1986; 293: 917-922.

17. Norhammar A, Tenerz A, Nilsson G, Hamsten A, Efendic S, Ryden $\mathrm{L}$, et al. Glucose metabolism in patients with acute myocardial infarction and no previous diagnosis of diabetes mellitus: A prospective study. Lancet 2002; 359: 2140-2144.

18. Tenerz A, Norhammar A, Silveira A, Hamsten A, Nilsson G, Ryden $\mathrm{L}$, et al. Diabetes, insulin resistance, and the metabolic syndrome in patients with acute myocardial infarction without previously known diabetes. Diabetes Care 2003; 26: 2770-2776.

19. Halter JB, Beard JC, Porte D Jr. Islet function and stress hyperglycemia: Plasma glucose and epinephrine interaction. Am J Physiol 1984; 247: E47-E52

20. Shamoon H, Hendler R, Sherwin RS. Altered responsiveness to cortisol, epinephrine, and glucagon in insulin-infused juvenile-onset diabetics: A mechanism for diabetic instability. Diabetes 1980; 29: $284-291$.

21. Uusitupa MI, Mustonen JN, Airaksinen KE. Diabetic heart muscle disease. Ann Med 1990; 22: 377-386.

22. Miyazaki C, Takeuchi M, Yoshitani H, Otani S, Sakamoto K, Yoshikawa J. Optimum hypoglycemic therapy can improve coronary flow velocity reserve in diabetic patients: Demonstration by transthoracic doppler echocardiography. Circ J 2003; 67: 945-950.

23. Tamada H, Nishikawa H, Mukai S, Setsuda M, Nakamura M, Suzuki $\mathrm{H}$, et al. Impact of diabetes mellitus on angiographically silent coronary atherosclerosis. Circ J 2003; 67: 423-426.

24. Tosaki A, Engelman DT, Engelman RM, Das DK. The evolution of diabetic response to ischemia/reperfusion and preconditioning in isolated working rat hearts. Cardiovasc Res 1996; 31: 526-536.

25. Iwakura K, Ito H, Ikushima M, Kawano S, Okamura A, Asano K, et al. Association between hyperglycemia and the no-reflow phenomenon in patients with acute myocardial infarction. J Am Coll Cardiol 2003; 41: $1-7$.

\section{Appendices}

JACSS Principal Investigators (*chairperson)

* Ogawa H (Kumamoto University), Asada Y (Miyazaki Medical College), Tei C (Kagoshima University), Kimura K (Yokohama City University Medical Center), Tsuchihashi K (Sapporo Medical University), Ishihara M (Hiroshima City Hospital), Miyazaki S, Yamagishi M, Ikeda Y (National Cardiovascular Center), Shirai M (Yamaguchi University), Hiraoka H (Osaka University), Inoue T (Oita National Hospital), Sonoda M (National Hospital Kyusyu Cardiovascular Center), and Saito F (Nihon University Surugadai Hospital).

JACSS Participating Institutions and Clinical Investigators

Honda T (Social Welfare Organization Imperial Gift Foundation Incorporated Saiseikai Kumamoto Hospital), Ogata Y (Japanese Red Cross Kumamoto Hospital), Saito T (Kumamoto Central Hospital), Hokamura Y (Kumamoto City Hospital), Mizuno Y (Kumamoto Kinoh Hospital), Miyagi H (Kumamoto National Hospital), Matsumura T (Labor Welfare Corporation Kumamoto Rosai Hospital), Tabuchi T (Yatsushiro Health Insurance General Hospital), Sakaino N (Amakusa Medical Center), Kimura K (Arao City Hospital), Obata K (Health Insurance Hitoyoshi General Hospital), Shimomura H (Fukuoka Tokushukai Medical Center), Matsuyama K (Social Insurance Ohmuta-Tenryoh Hospital), Nakamura N (Shinbeppu Hospital), Yamamoto N (Miyazaki Prefectural Nobeoka Hospital), Hase M (Sapporo Medical University School of Medicine), Matsuki T (Shinnittetsu Muroran General Hospital), Hashimoto A (Kushiro City General Hospital), Abiru M (Oji General Hospital), Matsuoka T (National Hospital Kyusyu Cardiovascular Center), Toda H, Ri S (Kagoshima City Hospital), Toyama Y, Yamaguchi H, Toyoshima S (Nanpuh Hospital), Torii H (Kagoshima Medical Association Hospital), Atuchi Y, Miyamura A (Tenyokai Chuo Hospital), Hamasaki S (Kagoshima University Faculty of Medicine), and Miyahara K (Shinkyo Hospital). 